ATLAS Internal Note

LARG-NO-057

10 December 1996

\title{
Design and Development of Tungsten-Liquid Argon Modules for the ATLAS Forward Calorimeter System
}

\author{
J. K. Mayer, R. S. Orr \\ Department of Physics and Astronomy, University of British Columbia \\ Vancouver, B.C., Canada \\ G. Oakham, M. O’Neill \\ Centre for Research in Particle Physics, Carleton University \\ Ottawa, Ontario, Canada \\ J. Dubeau \\ Department of Physics, Carleton University \\ Ottawa, Ontario, Canada \\ G. G. Stairs \\ Department of Physics, University of Toronto \\ Toronto, Ontario, Canada
}

Proceedings of the 5th International Conference on Advanced Technology and Particle Physics, Como, Italy, 7-11 October, 1996

\begin{abstract}
The design of a tungsten-liquid argon calorimeter for the high $|\eta|$ region of the ATLAS detector at the LHC is discussed. A novel design using hexagonally packed, tubular, thin gap electrodes running parallel to the beam direction is employed. The absorber matrix is composed of small "slugs" of sintered tungsten alloy. To test the mechanical design and construction methodology, a small engineering prototype, or "maquette", has been constructed. This maquette is a full featured, full depth device, deviating from the final production modules only in lateral size.
\end{abstract}




\section{INTRODUCTION}

The ATLAS experiment [1] employs liquid argon calorimetry in the region $1.5<|\eta|<4.7$. This $\eta$ coverage is spanned by two calorimeter systems. At lower $|\eta|$, a lead accordion electromagnetic section [2] is followed by a copper plate hadronic endcap calorimeter [3]. Beyond $|\eta|=3.2$, the high energies and rates are unsuitable for the plate design. Devices must be fast to keep pile-up

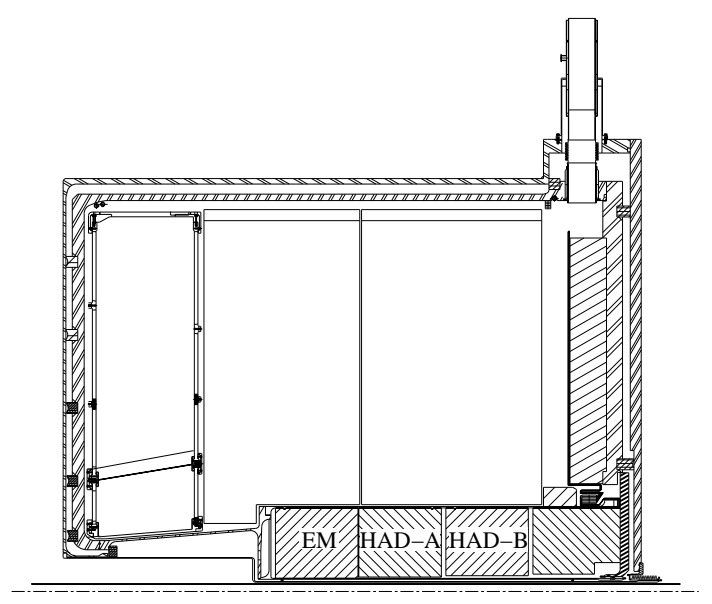

Figure 1: A quadrant of the endcap cryostat, showing the FCAL modules (EM, HAD-A, HAD-B) inside the endcap calorimeters.

effects low and must be designed to reduce the effects of positive ion buildup in the liquid argon. To meet these challenges, the ATLAS experiment has chosen to use a liquid argon "spaghetti" forward calorimeter (FCAL) integrated into the same cryostat with the endcap calorimeters (see Fig. 1). The FCAL uses rods placed inside tubes to form cylindrical, thin ionization gap electrodes packed in a hexagonal arrangement parallel to the beam axis. The thin gaps ensure that the device is fast enough to meet the technical challenges in this region. This geometry also eases difficulties in the placement and shielding of the forward muon systems and beam line elements.

Each of the two forward calorimeter systems is divided longitudinally into three sections: an electromagnetic (EM) section, and two hadronic (HAD-A and HAD-B) sections (see Fig. 1). The purpose of the EM module is to measure the energy and position of $\gamma$ 's from $\pi^{0}$ decay and to make a precision angle measurement of hadronic showers near the beginning of the shower, where the transverse spreading is still small. The hadronic modules are designed to measure the total hadronic energy in the very limited space available, and the division into two sections is to provide a measurement of the longitudinal development of the shower. The absorber medium for the EM section will be copper to improve sampling uniformity and allow longitudinal spreading of showers. The performance of an EM prototype is discussed in [4]. The hadronic sections will be composed of tungsten to increase the number of interaction lengths of calorimeter inside the cryostat. The total depth of the FCAL system is $9.3 \lambda$ which is sufficient to ensure that the degradation of the energy resolution due to end leakage is well below the intrinsic physical backgrounds. 


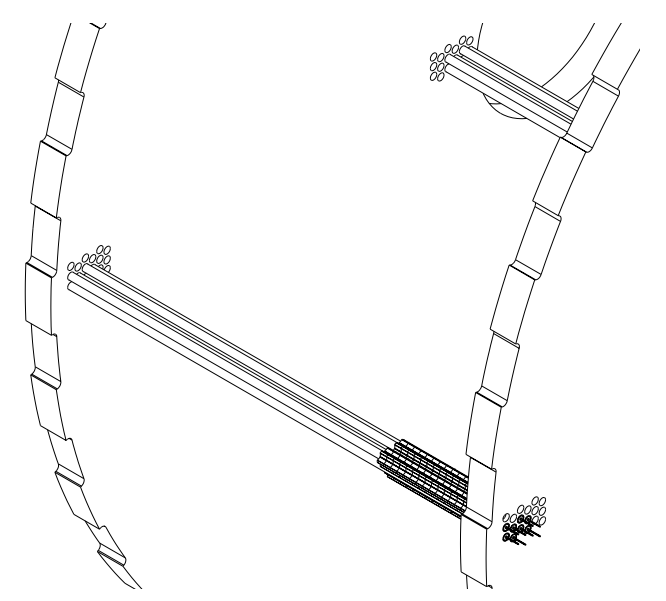

Figure 2: View of the HAD-A and HAD-B modules showing the assembled structure. Two $25 \mathrm{~mm}$ endplates are spanned by thin walled $\mathrm{Cu}$ tubes in a hexagonal pattern. The cutouts on the periphery of the endplates accommodate readout cables from FCAL modules further upstream.

\section{TECHNICAL CONSTRAINTS AND MODULE DESIGN}

The forward calorimeter system is in a region of the experiment where large fluxes of ionizing and neutron radiation are present. Yearly integrated ionization doses of $2,300 \mathrm{kGy}$ and neutron fluences of up to $10^{16} \mathrm{n} / \mathrm{cm}^{2}$ are expected when the LHC operates at full design luminosity [1]. The stability of materials under irradiation is a design concern. Mechanically the components must not deteriorate to the point where their functional use is compromised. Chemically, they must not add impurities to the liquid argon that trap electrons produced in the ionization process (for a discussion of this point, see [5]). As well, the production of long lived radioactive isotopes through neutron activation must be kept low. Buildup of these isotopes over many years of running could render the calorimeter modules unserviceable.

\begin{tabular}{|l|r|r|}
\hline Parameter & HAD-A & HAD-B \\
\hline Module Mass $(\mathrm{kg})$ & 3945 & 3723 \\
Inner radius $(\mathrm{mm})$ & 79 & 86 \\
Outer radius $(\mathrm{mm})$ & 456 & 456 \\
Length $(\mathrm{mm})$ & 450 & 450 \\
Electrode & & \\
Rod OD $(\mathrm{mm})$ & 4.75 & 5.5 \\
Tube ID $(\mathrm{mm})$ & 5.5 & 6.5 \\
Argon gap $(\mu \mathrm{m})$ & 375 & 500 \\
Spacing $(\mathrm{mm})$ & 8.18 & 9.00 \\
Depth $(\lambda)$ & 3.4 & 3.4 \\
No. of tubes & 10368 & 8128 \\
Tubes/readout cell & 6 & 8 \\
\hline
\end{tabular}

Table 1: HAD-A and HAD-B Design Parameters 
The FCAL HAD design uses only manifestly radiation hard materials: tungsten, copper, and quartz. Components which cannot be constructed from these materials, such as electronic readout boards, are made from polyimide plastics. Figure 2 shows the basic construction technique of the HAD-A and HAD-B modules. Table 1 lists some important parameters used for each module. Each FCAL hadronic module consists of two precision drilled copper plates, each $25 \mathrm{~mm}$ thick, with the copper electrode tubes extending between them. The chosen electrode structure consists of centreless-ground tungsten anode rods inside copper tubes, a geometry similar to that used in the EM FCAL modules [4]. Each hole in the endplates which is to accommodate the end of a tube has a small groove cut into it near its outer face, $1.0 \mathrm{~mm}$ wide and $150 \mu \mathrm{m}$ deep (see Fig. 3 ). The

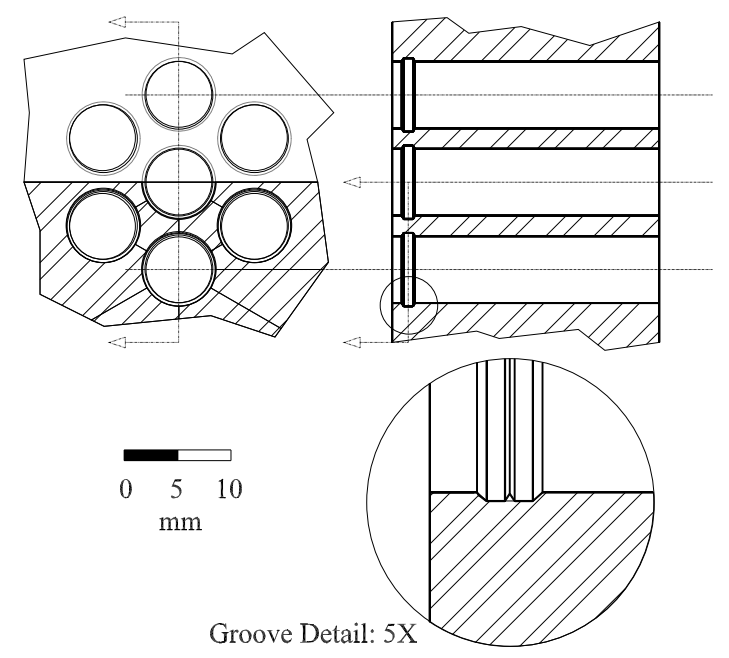

Figure 3: The endplate hole details showing the groove for tube swaging. The inverted V-notch inside each groove helps maintain electrical and mechanical reliability of the swage.

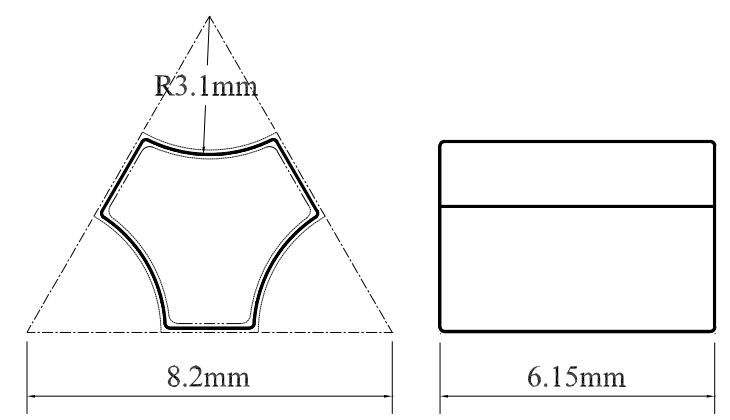

Figure 4: The size and shape of a HAD-A sintered tungsten alloy slug. The figure at the left depicts the nominal profile size of the slug (dark outline) enveloped by a $100 \mu \mathrm{m}$ tolerance limit (light outlines).

copper tubes are attached and locked to the endplates by radially deforming them into the grooves. 
A $50 \mu \mathrm{m}$ high inverted V-notch midway through the groove helps maintain good electrical and mechanical bonding. This swaging technique was developed to address the issues of cleanliness and ease of assembly. Early welding trials in copper produced unsuitable residues around the bonding area, and there was concern over the possible formation of small features at the entrance to the tube which could cause high voltage breakdown in the area. The swaging technique remains the baseline method while other welding techniques are studied. Tests performed on the endplate-tube swage indicated a bonding strength approaching the failure of the tube. The $\sim 10,000$ tube hexagonal structure provides the mechanical integrity of the module. The tungsten absorber matrix between the tubes is not part of the load bearing structure. In fact, a space of $1.25 \mathrm{~mm}$ is required between the endplates and the matrix to account for the larger thermal expansion coefficient of copper. When cooled to liquid argon temperatures, this space shrinks to $0.25 \mathrm{~mm}$.

Tungsten is difficult to form and machine, so innovative construction technologies must be used to produce the absorber matrix. Pure tungsten is too refractory and expensive to work with, so the HAD modules are assembled using sintered tungsten components. Sintered tungsten is formed by combining tungsten powder with a small percentage of other metals and an organic binder into "greenform" pieces approximately $20 \%$ larger than the specified dimensions of the final parts. The organic binder is added to give support to the greenforms and is completely burned off during the subsequent sintering operation. The parts are fired at the melting temperature of the binder metals, causing the parts to shrink as the metal flowed between the $\mathrm{W}$ granules. Therefore, with only modest heating, this powder mixture can be used to form complex and relatively easily machinable parts. Depending on the percentages of the binder metals used, the density of the sintered alloy can range from $16.0-18.5 \mathrm{~g} / \mathrm{cm}^{3}$. Industrial "pure" tungsten has a density of $19.0 \mathrm{~g} / \mathrm{cm}^{3}$.

The calorimeter's absorber matrix is formed by inserting sintered tungsten slugs between the copper tubes. Figure 4 shows the dimension and shape of the pieces. The slugs are pre-formed

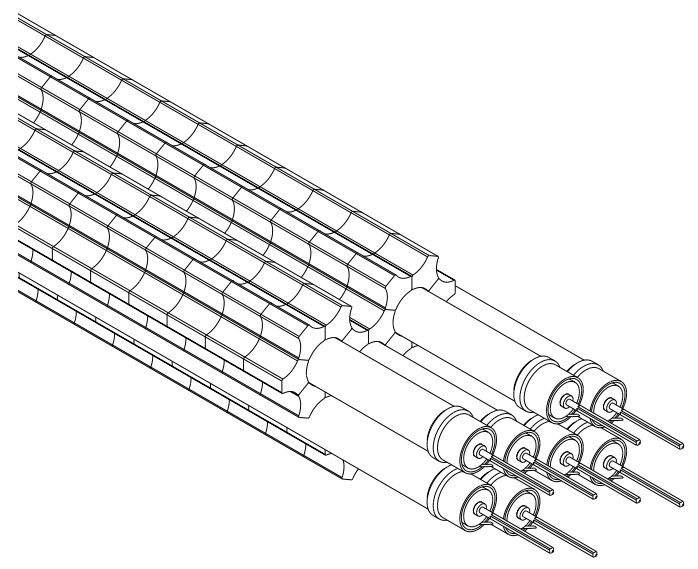

Figure 5: A cut-away view of the tubular electrodes embedded in a matrix of sintered W slugs. The slugs end where the endplate (not shown) starts. Posts are staked into the ends of the W rods for electrical connections. Also shown are the tube swages located near the ends of the electrodes. The position of these swages correspond to the grooves in the endplate holes.

before sintering to be of the appropriate shape to fit into the hexagonal matrix (Fig. 5). The length of the slug is constrained by the capabilities of industry to maintain the tolerance on their straightness. The slugs must be precisely shaped so that all gaps are small. 
Once the endplates, tubes and slugs are assembled, pure tungsten rods are inserted down the tubes to complete the electrodes. Pure tungsten is used in this instance as the rods are a standard, industrially produced item. A line of polyimide coated quartz fibre is spiraled around the rods to keep them centred. Connection to high voltage and readout electronics is made via pins staked into the end of each rod. Small polyimide washers are placed over the rods to retain them in place and to prevent the quartz fibre from unwinding.

Due to the large radiation fields present in the FCAL region, the uninstrumented areas around the modules must be filled with material to improve radiation shielding. Because of the hexagonal packing of the electrodes, there is an irregular arrangement of tubes at both the inner and outer circular periphery of the device. As well, the electrodes of the HAD-A and HAD-B modules are ganged together into groups of six and eight respectively; these then form the smallest cells read out from the calorimeter. This ganging and the hexagonal packing makes it difficult to treat the peripheries in a simple manner. At the inner bore of the calorimeter, a layer of electrode tubes will retain the inner layer of $\mathrm{W}$ slugs. To make a smooth, cylindrical bore, a piece of sintered $\mathrm{W}$ alloy will be precisely machined to fit the pattern of the retaining tubes. At the outer edge of the modules, a similar technique will be employed using machined copper plates.

This design results in a very dense, radiation hard calorimeter with a very small sampling fraction. The mean density of the HAD-A and HAD-B modules are $14.8 \mathrm{~g} / \mathrm{cm}^{3}$ and $14.3 \mathrm{~g} / \mathrm{cm}^{3}$ respectively. This geometry also allows easy lateral division of the calorimeter into "towers" of appropriate size.

\section{THE FCAL TUNGSTEN MAQUETTE}

Because of the calorimeter's complexity and unique design, a small engineering model was constructed, the "maquette", to study and address a number of technical uncertainties. Prior to the construction of the maquette, there was no firm understanding of the capabilities of industrial manufacturers in producing the very precise components required. In particular, until a firm order of tungsten slugs was made, there was a large uncertainty as to their cost, the production success rate, and whether the required tolerances could be met. The maquette also served as a useful platform for understanding construction procedures and developing tooling to be used on subsequent prototypes and production modules. With over 4.5 million parts to be incorporated into the final four HAD modules, automated handling and assembly procedures will be required to construct them in a timely manner.

The maquette was designed as a laterally reduced HAD-A module and therefore incorporates all the features proposed for the production units. It consists of two $25 \mathrm{~mm}$ thick copper endplates with thin-walled $(250 \mu \mathrm{m})$ tubes swaged into them, giving a total length of $450 \mathrm{~mm}$. The lateral dimensions of the maquette were specified to account for the readout structure of the HAD-A modules and to allow for good statistical sampling of components. The HAD-A electrodes are connected together into readout cells of six. Therefore the maquette was constructed to accommodate 108 sensitive electrodes (18 readout cells), with a peripheral layer of tubes acting as slug retainers (see Fig. 6). With this lateral size, a good sampling of the manufactured components was possible. The large number of electrodes also facilitated studying variations due to tolerance fluctuations and component quality.

\subsection{Maquette Components}

Prior to the construction of the maquette, the component with the largest technological uncertainty was the sintered tungsten alloy slugs. It was not certain that these could be produced to the 


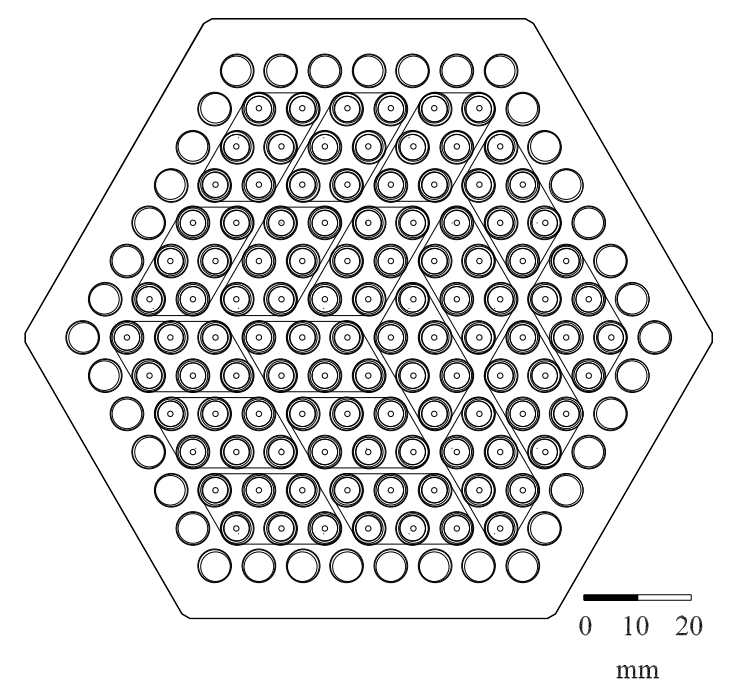

Figure 6: The shape and size of the maquette endplates. Individual electrodes are ganged into groups of six, forming 18 readout cells in the centre of the maquette. One layer of tubes around the periphery retains the outer layers of slugs.

tolerances required to maintain the high density of the absorber matrix. Slugs were produced using a powder mixture of $97 \% \mathrm{~W}, 2.1 \% \mathrm{Ni}$, and $0.9 \%$ Fe to produce sintered slugs having a density of $18.5 \mathrm{~g} / \mathrm{cm}^{3}$. More than 16,000 slugs of length $6.15 \mathrm{~mm}$ were required to construct the maquette. Their lateral profile size was checked using a gauge and was found to meet the tolerance on maximum size. To check compliance on minimum lateral dimensions, the mass, density, and lengths of a sample of slugs was measured. This showed that they conformed to the specified dimensions and tolerance limits. Their density was determined to be $18.5 \mathrm{~g} / \mathrm{cm}^{3}$, as required.

A sample of slugs was irradiated with neutrons at the University of Toronto Slowpoke Reactor Facility. A fluence of $10^{13} \mathrm{n} / \mathrm{cm}^{2}$ was used to search for impurities present in the $\mathrm{W}$ alloy, most notably cobalt, which could activate over time and render the calorimeter unserviceable. A large percentage of commercially available tungsten powder is reclaimed from machine tools that have been alloyed with cobalt to increase hardness. These studies indicated impurities were present, but at an acceptable level below 5 ppm. This testing will be a continuing qualification process as materials arrive for the production of subsequent modules.

To maximize the volume of the tungsten slugs, and therefore the overall density of the modules, the copper electrode tubes that span the endplates were specified to have thin walls of $250 \mu \mathrm{m}$. Measurements on the manufactured tubes indicated that the mean inner diameter (ID) was $26 \mu \mathrm{m}$ smaller than expected. However, the r.m.s. fluctuation of the ID was $6 \mu \mathrm{m}$, better than the $10 \mu \mathrm{m}$ tolerance. Therefore only a slight adjustment in the manufacturing process should be required to produce the tubes at specified dimensions for future modules.

Pure tungsten rods of diameter $4.75 \mathrm{~mm}$ were specified for the maquette. Tolerance checks of the manufactured rods showed excellent compliance, with an r.m.s. fluctuation on the diameter of $4 \mu \mathrm{m}$, much better than the $10 \mu \mathrm{m}$ tolerance specified. A small, $1.40 \mathrm{~mm}$ hole was drilled in one end of each rod. This accommodated a staked wire wrap terminal to be used for high voltage and signal connections. 


\section{$3.2 \quad$ Assembly}

The matrix was constructed by jigging the endplates $400 \mathrm{~mm}$ apart and assembling alternate layers of tubes and slugs, starting at the bottom and working upwards. To avoid systematically pulling the endplates together during swaging, tubes were initially only locked at one end. Once the entire matrix was stacked, two additional jig plates were attached before the second swage was made. Insertion of the tungsten rods was the final assembly stage. A nose piece was fitted over the lead end of the rod, trapping one end of the quartz fibre. The rod was twisted as it was loaded into the tube to spiral the fibre around it. Once in place, the nose piece was removed, and a fibre retainer attached.

The assembly of the maquette provided important and very useful information on tooling and assembly procedures. A rudimentary, yet effective, slug loading mechanism was developed. This will form the basis of a design for a vibratory feeder for the next modules. Much time and effort was spent in developing a tool which would reliably and consistently swage the copper tubes into the endplates. Practical experience of the swaging process has helped in understanding how to improve the process for the future. Loading of the tungsten rods into the tubes was done by hand for the maquette, but it has indicated where the difficulties will lie in designing a semi-automated insertion machine.

\section{FUTURE STUDIES}

The production of the maquette tested the manufacturing capabilities of suppliers and has shown where there are difficulties and room for improvement. It also provided assembly experience to help in the design of tooling for the production modules. Most important, it showed that the design of the tungsten hadronic forward calorimeter modules is sound.

A number of studies on the completed maquette are currently underway. The mechanical behaviour of the device in liquid argon is being studied. The uniformity of the electrical properties of the electrodes and the consistency of these properties under temperature cycling is also being studied.

In addition to tests on the maquette, we are working closely with tungsten manufacturers to see if longer slugs can be produced. Longer slugs would ease handling and assembly, and reduce gaps due to tolerance buildups, leading to an increase in overall density.

\section{ACKNOWLEDGMENTS}

We would like to thank P. Gravelle and P. Miller for all their efforts and their technical input during construction of the maquette. As well we would like to thank E. Hayashi and T. Karmo for all their assistance. We would also like to thank J. Rutherfoord and L. Shaver of the University of Arizona for fruitful discussions and suggestions.

This work was supported by the Natural Sciences and Engineering Research Council of Canada.

\section{References}

[1] The ATLAS Collaboration, Technical Proposal for a General Purpose pp Experiment at the Large Hadron Collider at CERN, CERN/LHCC/94-43 (1994).

[2] A. Chekhtman, D. Fouchez and E. Monnier, ATLAS Internal Note LARG-NO-4 (1994). 
[3] T. Hodges et al., ATLAS Internal Note CAL-NO-60 (1994).

[4] J. Armitage et al., to appear in the proceedings of the 5th International Conference on Advanced Technology and Particle Physics, Como, Italy, October 7-11, 1996.

[5] W. Hofmann et al., Nucl. Inst. Meth. 135 (1976) 151- 156. 